\title{
Adaptive low-rank channel estimation for multi- band OFDM ultra-wideband communications
}

\author{
Chia-Chang $\mathrm{Hu}^{*}$ and Shih-Chang Lee
}

\begin{abstract}
In this paper, an adaptive channel estimation scheme based on the reduced-rank (RR) Wiener filtering (WF) technique is proposed for multi-band (MB) orthogonal frequency division multiplexing (OFDM) ultra-wideband (UWB) communication systems in multipath fading channels. This RR-WF-based algorithm employs an adaptive fuzzy-inference-controlled (FIC) filter rank. Additionally, a comparative investigation into various channel estimation schemes is presented as well for MB-OFDM UWB communication systems. As a consequence, the FIC RR-WF channel estimation algorithm is capable of producing the bit-error-rate (BER) performance similar to that of the full-rank WF channel estimator and superior than those of other interpolation-based channel estimation schemes.
\end{abstract}

Keywords: channel estimation, MB-OFDM, ultra-wideband (UWB), Wiener filter

\section{Introduction}

Ultra-wideband (UWB) wireless systems have generated considerable interest as an indoor short-distance highdata-rate transmission in wireless communications over the past few years. A number of promising advantages, such as low power consumption, low cost, low complexity, noise-like signal, resistant to dense multipath and jamming, and excellent time-domain resolution, have made UWB systems perfectly suitable for personal computing (PC), consumer electronics (CE), mobile applications, and home entertainment networks. Applications of UWB radio techniques to short-range wireless communications, such as sensor networks and wireless personal area networks (WPANs), are currently being explored [1]. Two competing UWB technologies for physical layer (PHY) of the WPANs are investigated by the IEEE 802.15.3a standards task group (TG3a) [2]. One is the direct-sequence (DS) UWB link scheme and the other is the multi-band (MB) orthogonal frequency division multiplexing (OFDM) UWB system.

The MB-OFDM UWB communication systems [3] have recently drawn extensive attention due to potential for providing high data rate under a low transmission power. The MB-OFDM developed by the WiMedia Alliance [4] is the first UWB radio transmission technology

\footnotetext{
* Correspondence: ieecch@ccu.edu.tw

Department of Communications Engineering, National Chung Cheng

University 168 University Road, Min-Hsiung, Chia-Yi 621, Taiwan
}

to obtain international standardization. This promising wireless-connectivity technique increases successfully both the traffic capacity and the frequency diversity. In MB-OFDM UWB wireless systems, by utilizing several types of time-frequency codes (TFCs) in the preamble part, multiple users are allowed to use the same frequency-band group simultaneously to provide frequency diversity as well as channelization and multiple-access capability among different piconets. That is the primary reason why the preamble symbols gain a high probability of being corrupted by multiple-access interference (MAI). To enhance the system performance, pilotassisted channel estimation schemes are commonly employed for the MB-OFDM UWB systems. In particular, the performance of channel estimation in a pilotaided MB-OFDM UWB system has been investigated based on the least-squares (LS) algorithm [5], the maximum likelihood estimator (MLE) [6], and the minimum mean-square error (MMSE) estimator [5,7]. The channel estimation with the use of the MLE obviates the necessity of the information of either the channel statistics or the operating signal-to-noise ratio (SNR). However, it is already known that the computational costs for these estimators are very expensive and thus lead to a limited usage in practice. This requirement is, in general, prohibitive for low-power and cost-effective wireless UWB devices.

\section{Springer}

(C) $2011 \mathrm{Hu}$ and Lee; licensee Springer. This is an Open Access article distributed under the terms of the Creative Commons Attribution License (http://creativecommons.org/licenses/by/2.0), which permits unrestricted use, distribution, and reproduction in any medium, provided the original work is properly cited. 
In this paper, an adaptive low-rank channel estimation scheme based on the Wiener filtering (WF) technique is proposed for MB-OFDM UWB communication systems. This reduced-rank (RR) WF-based algorithm employs an adaptive 2-to-1 fuzzy-inference controlled (FIC) filter rank. It can be shown that the fuzzy-inference system (FIS) [8] offers an effective and robust means to monitor instantaneous fluctuations of a dense multipath channel and thus is able to assist the RR-WF-based channel estimator in selecting an appropriate time-varying filter rank $p$. As a result, the proposed RR-WF-based channel estimation possesses the potential to accomplish substantial saving on computational complexity without affecting system bit-error-rate (BER) performance. To emphasize the importance of the use of an adaptive RRWF scheme, both the MSE and the BER performances are evaluated and compared with the piecewise linear [9], the Gaussian second-order [10], the cubic-spline [10], the LS, and the fullrank WF channel estimation [5] algorithms. Simulation results have shown that the proposed FIC RR-WF scheme reduces successfully computational complexity without sacrificing the BER performance under different UWB channel conditions.

The remainder of this paper is organized as follows. In Section 2, a brief introduction of the MB-OFDM UWB system architecture and channel model is presented. The reduced-rank Wiener filter channel estimation scheme is developed in Section 3. Principles of the 2-to1 fuzzy-inference-determined filter-rank selection mechanism are introduced in Section 4. Section 5 analyzes the computational complexity of the 2-to-1 FIC filter-rank selection scheme. Simulation results are compared and analyzed in Section 6. Finally, some concluding remarks are drawn in Section 7.

\section{MB-OFDM UWB SYSTEM MODEL}

In an MB-OFDM UWB system, the spectrum from 3.1 $\mathrm{GHz}$ to $10.6 \mathrm{GHz}$ is divided into 14 sub-bands with a bandwidth of $528 \mathrm{MHz}$ each, and the data are transmitted across these sub-bands using a specific TFC [3]. The system operates in one sub-band and then switches to another sub-band after a short time. In each subband, the OFDM modulation scheme is used to transmit data symbols. The transmitted symbols are time-interleaved across the sub-bands to utilize the spectral diversity in order to improve the transmission reliability. Additionally, it is important to note that depending on the selected TFC, the MB-OFDM system is equipped with the frequency-hopping $(\mathrm{FH})$ control mechanism. The feature of the FH pattern controlled by the TFCs enables multiple simultaneously operating piconets (SOPs) at the same band group. However, this is of little impact on the channel estimation since it is assumed that each sub-band is estimated independently. The fundamental transmitter and receiver structure of an MB-OFDM system is illustrated in Figure 1. At the transmitter of an MB-OFDM system, the bits from information sources are first mapped to quadrature phase-shift keying (QPSK) symbols. To exploit time-frequency diversity and combat multipath fading, the coded bits are interleaved according to some preferred time-frequency patterns, and the resulting bit sequence is mapped into constellation symbols and then converted into the $l$ th OFDM block of $N$ symbols $X(l, 0)$, $X(l, 1), \ldots, X(l, N-1)$ by the serial-to-parallel converter. The $N$ symbols are the frequency components to be transmitted using the $N$ subcarriers of the OFDM modulator and are converted to OFDM symbols $x(l, 0), x(l$, $1), \ldots, x(l, N-1)$ by the unitary inverse fast Fourier transform (IFFT), i.e.

$$
\begin{aligned}
x(l, n) & =\operatorname{IFFT}\{X(l, k)\} \\
& =\frac{1}{N} \sum_{k=0}^{N-1} X(l, k) e \frac{j 2 \pi k n}{N}, \quad n=0,1, \ldots, N-1 .
\end{aligned}
$$

A cyclic prefix (CP) of length $P(P \leq N)$ is added to the IFFT output to eliminate the intersymbol interference caused by the multipath propagation. The resulting $N+P$ symbols are converted into a continuous-time baseband signal $x(t)$ for transmission.

The UWB channel model proposed for the IEEE 802.15.3a standard is considered [11]. The multipath UWB channel impulse response can be expressed as

$$
h(t)=\chi \sum_{j=1}^{J} \sum_{d=1}^{D} \alpha_{d, j} \delta\left(t-T_{j}-\tau_{d, j}\right),
$$

where $\chi$ represents the lognormal shadowing factor of propagation channels, $\delta(t)$ is the Dirac delta function, $T_{j}$ denotes the delay of the $j$ th cluster's first path, $\alpha_{d, j}$ is the multipath gain coefficient and $\tau_{d, j}$ is the delay of the $d$ th multipath component (ray) relative to the $j$ th cluster arrival time $T_{j}, J$ is the cluster number, and $D$ is the multipath number in a cluster. Based on the SalehValenzuela (S-V) model [11-13] and the measurements of actual channel environments, four types of indoor multipath channels, namely CM1, CM2, CM3, and CM4, are defined by the WiMedia Alliance with different values for parameters [4]. In particular, the IEEE 802.15 standard model assumes that the channel stays either completely static or changes completely from one data burst to the next. In other words, the time variations (coherence time) of the channel are not considered since most of applications are targeted for high-data-rate communications in slowly fading indoor environments, such as pedestrian speeds or slower $[4,13]$. With a choice of the CP length greater than the maximum delay spread of the UWB channel [4], OFDM allows for 


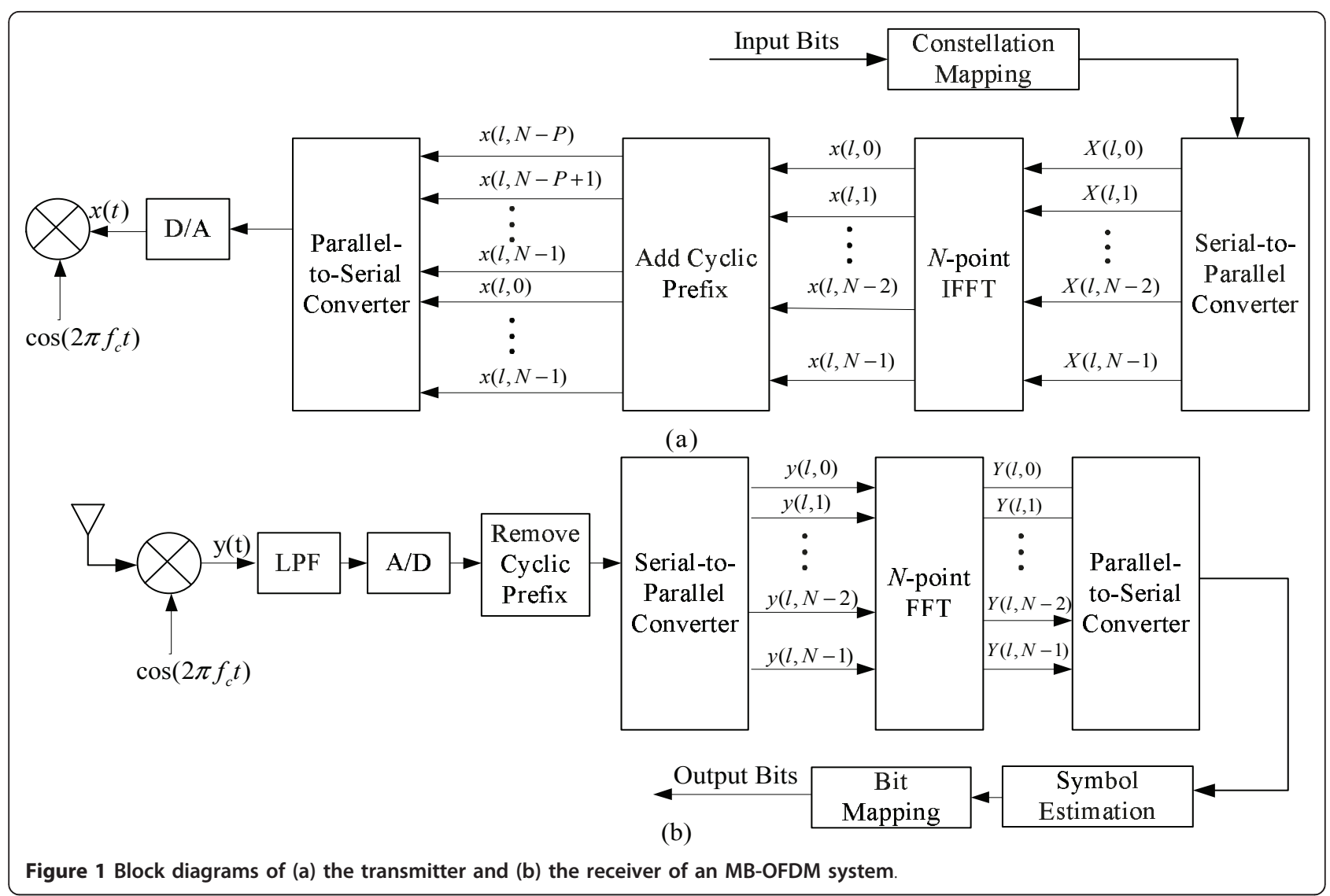

each UWB sub-band to be divided into a set of $N$ orthogonal narrowband channels. In such conditions, the intersymbol interference (ISI) can be effectively suppressed, and thus, sufficient multipath energy is captured to make the impact of the intercarrier interference (ICI) minimized. Therefore, perfect frequency synchronization is assumed, and the ICI is negligible in what follows. Furthermore, it is important to notice that in the presence of ICI due to the high delay and Doppler spread, dedicated ICI mitigation algorithms [14-17] are required to suppress the ICI over fast "time-varying" fading channels.

The UWB channel in the discrete time domain is modeled as a $N_{h}$-tap finite-impulse-response (FIR) filter whose impulse response of the $l$ th OFDM block on a sub-band is denoted by

$$
\mathbf{h}(l)=\left[h(l, 0), h(l, 1), \ldots, h\left(l, N_{h}-1\right)\right]^{\top},
$$

where $(\cdot)^{\top}$ denotes the transposition operation. The corresponding channel frequency responses

$$
\mathbf{H}(l)=[H(l, 0), H(l, 1), \ldots, H(l, N-1)]^{\top} .
$$

are given by $\mathbf{H}(l)=\mathbf{F}_{N_{h}} \mathbf{h}(l)$, where is the first $N_{h}$ columns of the $N$-point DFT matrix. For channel estimation, a total of $N_{p}$ pilot signals are uniformly inserted into the transmitted OFDM symbols at known locations $\left\{i_{n}: 1 \leq n \leq N_{p}\right\}$. Let

$$
\mathbf{X}_{p}(l)=\operatorname{diag}\left\{X\left(l, i_{1}\right), X\left(l, i_{2}\right), \ldots, X\left(l, i_{N_{p}}\right)\right\},
$$

denote the $N_{p} \times N_{p}$ matrix containing the FFT output of the $l$ th OFDM block at the pilot subcarriers. At the demodulator, after removing the cyclic prefix, the unitary FFT is performed on the remaining $N$ symbols to obtain

$$
\mathbf{Y}(l)=\mathbf{X}(l) \mathbf{H}(l)+\mathbf{W}(l),
$$

where $\mathbf{X}(l)=\operatorname{diag}\{X(l, 0), X(l, 1), \ldots, X(l, N-1)\}$ in (6) stands for the transmitted data symbol, $\mathbf{Y}(l)=[Y(l, 0), Y$ $(l, 1), \ldots, Y(l, N-1)]^{\top}$ represents the received data symbol, $\mathbf{H}(l)$ as in (4) indicates the channel frequency response, and $\mathbf{W}(l)=[W(l, 0), W(l, 1), \ldots, W(l, N-1)]^{\top}$ denotes the additive noise component, of the $l$ th OFDM block.

\section{Reduced-rank Wiener filter channel estimation}

The Wiener filter (WF) estimator [5] employs the second-order statistics of the channel conditions to minimize the MSE. The WF yields much better performance 
than the LS-based estimator, especially under the low SNR scenarios. A major drawback of the WF estimator is its high computational complexity, especially if matrix inversion operation is required each time as the data in the transmitted vector are altered. The WF estimation of $\mathbf{H}(l)$ [5] can be obtained as

$$
\hat{\mathbf{H}}_{\mathrm{WF}}(l)=\mathbf{R}_{\mathbf{H}(l) \mathbf{H}(l)}\left\{\mathbf{R}_{\mathbf{H}(l) \mathbf{H}(l)}+\sigma_{w}^{2}\left[\mathbf{X}(l) \mathbf{X}^{\mathrm{H}}(l)\right]^{-1}\right\}^{-1} \hat{\mathbf{H}}_{\mathrm{LS}}(l),
$$

where $(\cdot)^{\mathrm{H}}$ means the conjugate transpose operation, $\sigma_{w}^{2}$ is the variance of the AWGN, $\mathbf{R}_{\mathbf{H}(l)} \mathbf{H}(l)$ denotes the auto-covariance matrix of the channel, given by $\mathbf{R}_{\mathbf{H}(l) \mathbf{H}}$ $(l) \triangleq E\left\{\mathbf{H}(l) \mathbf{H}^{\mathrm{H}}(l)\right\}$, and the LS estimator of $\mathbf{H}(l)[5]$ is $\hat{\mathbf{H}}_{\mathrm{LS}}(l)=\mathbf{X}^{-1}(l) \mathrm{Y}(l)=\left[\frac{Y(l, 0)}{X(l, 0)}, \frac{Y(l, 1)}{X(l, 1)}, \ldots, \frac{Y(l, N-1)}{X(l, N-1)}\right]^{\top}$. The computation of the WF-estimated channel transfer function requires the matrix inversion operation. A simplified WF estimation is obtained by averaging over the transmitted data to avoid the inverse matrix operation [18], and then Eq.(7) can be simplified as

$$
\hat{\mathbf{H}}_{\mathrm{WF}}(l)=\mathbf{R}_{\mathbf{H}(l) \mathbf{H}(l)}\left(\mathbf{R}_{\mathbf{H}(l) \mathbf{H}(l)}+\frac{\beta}{\mathrm{SNR}} \mathbf{I}\right)^{-1} \hat{\mathbf{H}}_{\mathrm{LS}}(l),
$$

where

$$
\begin{aligned}
& \mathrm{SNR}=\frac{E\left\{|X(l, k)|^{2}\right\}}{\sigma_{w}^{2}}, \\
& \beta=E\left\{|X(l, k)|^{2}\right\} E\left\{\left|\frac{1}{X(l, k)}\right|^{2}\right\} .
\end{aligned}
$$

Here, $\beta$ is a constant of the constellation used for the signal mapper, $\mathbf{I}$ is an identity matrix, and $|\cdot|$ indicates the absolute value. To reduce the computational complexity, a low-rank approximation by using singular value decomposition (SVD) [18] is adopted. This scheme reduces the rank of $\mathbf{R}_{\mathbf{H}(l) \mathbf{H}(l)}$ up to a threshold level $p$. The SVD of $\mathbf{R}_{\mathbf{H}(l) \mathbf{H}(l)}$ is performed as follows:

$$
\mathbf{R}_{\mathbf{H}(l) \mathbf{H}(l)}=\mathbf{U} \boldsymbol{\Lambda} \mathbf{U}^{\mathrm{H}},
$$

where $\mathbf{U}$ is the decomposed unitary matrix from $\mathbf{R}_{\mathbf{H}(l)}$ $\mathbf{H}(l)$ containing the singular vectors and $\boldsymbol{\Lambda}$ is a diagonal matrix containing the singular values $\lambda_{0} \geq \lambda_{1} \geq \ldots \geq \lambda_{N-1}$ on its diagonal. Then, substituting (11) into (8) derives Eq.(12) given by

$$
\hat{\mathbf{H}}_{\mathrm{WF}}(l)=\mathbf{U} \boldsymbol{\Lambda}\left(\boldsymbol{\Lambda}+\frac{\beta}{\mathrm{SNR}} \mathbf{I}\right)^{-1} \mathbf{U}^{\mathrm{H}} \hat{\mathbf{H}}_{\mathrm{LS}}(l) .
$$

Subsequently, the rank-reduction technique applied for the WF estimation is given as follows:

$$
\hat{\mathbf{H}}_{\mathrm{RR}-\mathrm{WF}}(l)=\mathbf{U} \boldsymbol{\Delta}_{p} \mathbf{U}^{\mathrm{H}} \hat{\mathbf{H}}_{\mathrm{LS}}(l),
$$

Where $\Delta_{p}$ is a diagonal matrix containing the values

$$
\delta_{k}=\left\{\begin{aligned}
\frac{\lambda_{k}}{\lambda_{k}+\frac{\beta}{\mathrm{SNR}}}, k & =0,1, \ldots, p-1, \\
0, k & =p, p+1, \ldots, N-1 .
\end{aligned}\right.
$$

\section{Fuzzy-inference filter-rank selection}

The 2-to-1 fuzzy inference system (FIS) [8], based on the principle of fuzzy logic [19], uses the squared error $\left(e^{2}(l)\right)$ and the squared error variation $\left(\Delta e^{2}(l)\right)$ as the input variables at OFDM block $l$ to assign the number of the filter rank $p(l+1)$. That is,

$$
p(l+1)=\operatorname{FIS}\left(e^{2}(l), \Delta e^{2}(l)\right),
$$

where

$$
e^{2}(l)=\frac{1}{N} \sum_{k=0}^{N-1}|H(l, k)-\hat{H}(l, k)|^{2},
$$

and

$$
\Delta e^{2}(l)=\left|e^{2}(l)-e^{2}(l-1)\right| .
$$

In essence, the basic configuration of the FIS comprises four essential procedures, namely (i) fuzzy sets for parameters, (ii) fuzzy control rules, (iii) fuzzy operators, and (iv) defuzzification processes, which map a twoinput vector, $\left(e^{2}(l), \Delta e^{2}(l)\right)$, into a single-output parameter $p$ for the adaptive time-varying filter-rank selection, as illustrated in Figure 2. Note that the input variables of a fuzzy logic system can be appropriately determined to include other types of parameters, such as duration of training, input power, and other useful variables $[8,20,21]$, which depend primarily on the applications in reality. Owing to the flexibility and richness of the FIS, it is able to produce many different mappings. The function of each procedure in the FIS is introduced briefly as follows:

\section{1) Fuzzy sets for parameters}

The input variables of the FIS are transformed to the respective degrees to which they belong to each of the appropriate fuzzy sets, via membership functions (MBFs). In what follows, the $\left(e^{2}, \Delta e^{2}\right)$-FIS system with the $(4,4)$-partitioned regions to the fuzzy I/O domains [8] is employed, due to its excellent performance and moderate complexity. The output of the fuzzification process demonstrates a fuzzy degree of membership between 0 and 1 . 


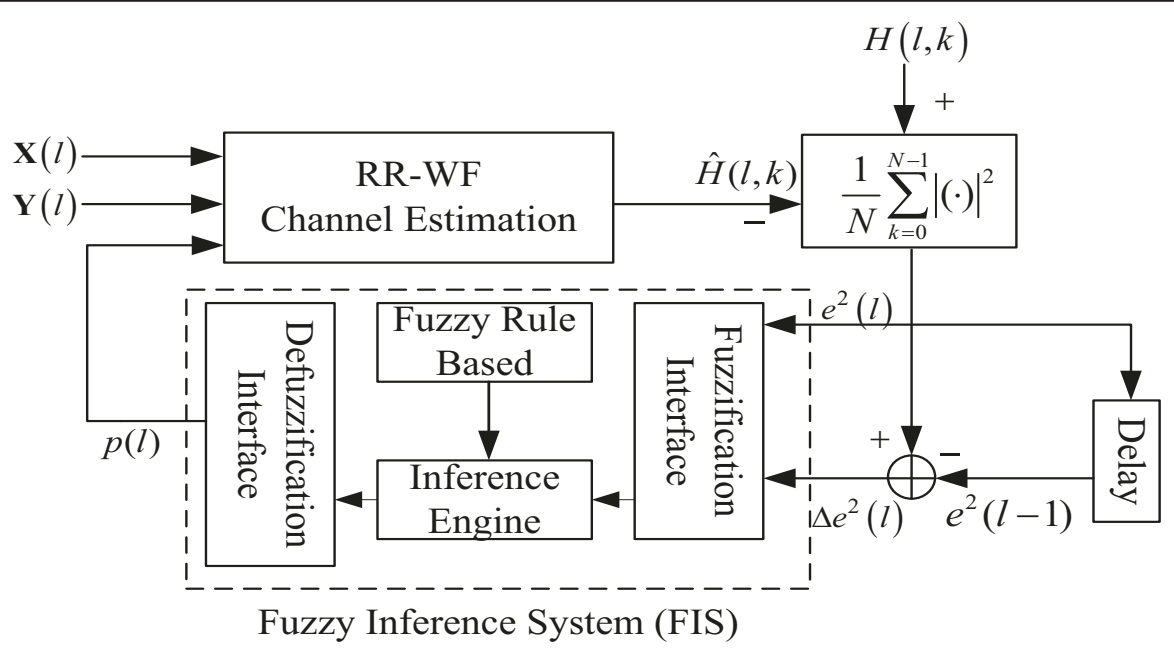

(a)

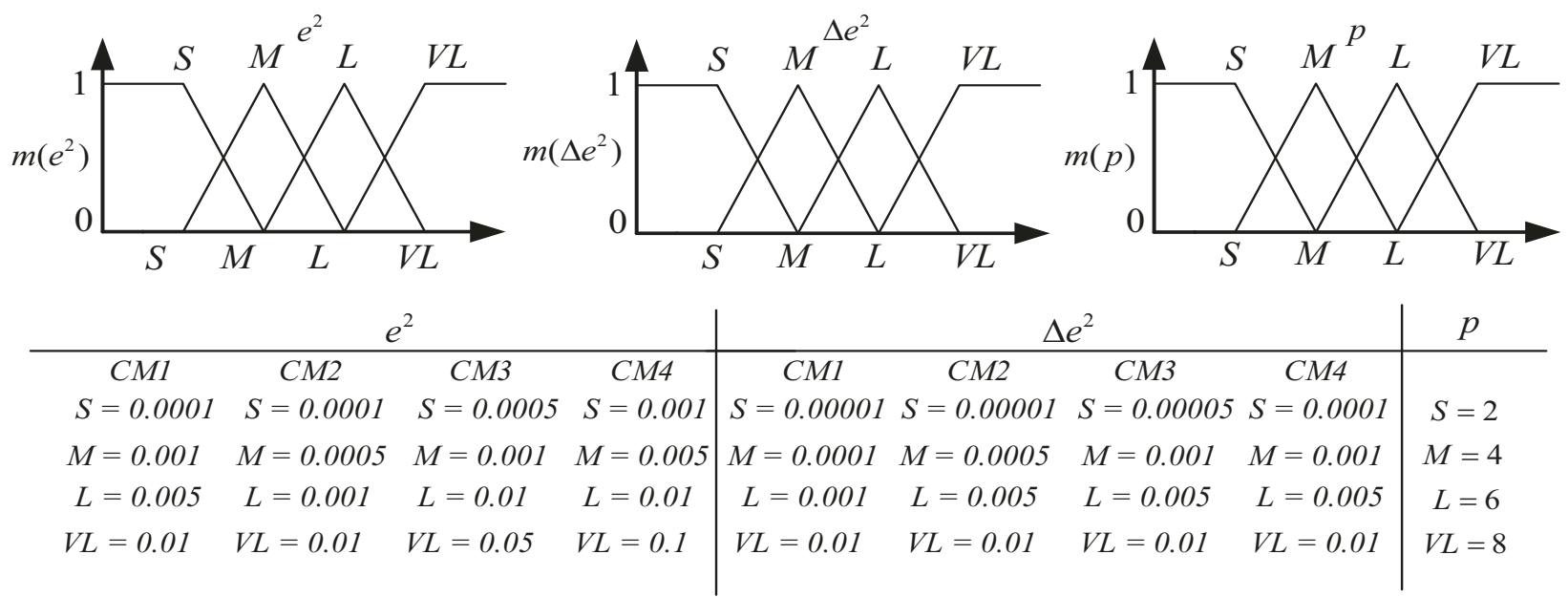

(b)

\begin{tabular}{rc|cccc} 
& \multicolumn{4}{c}{$e^{2}$} \\
& $p$ & $S$ & $M$ & $L$ & $V L$ \\
\hline$S$ & $S$ & $M$ & $M$ & $L$ \\
$\Delta e^{2}$ & $M$ & $S$ & $M$ & $L$ & $L$ \\
& $L$ & $M$ & $M$ & $L$ & $L$ \\
$V L$ & $L$ & $L$ & $L$ & $V L$
\end{tabular}

(c)

Figure 2 The fuzzy-inference-based variable filter-rank selection algorithm is illustrated by means of (a) block diagram, (b) three membership functions, and (c) predicate box, of the 2-to-1 fuzzy inference system.

\section{2) Fuzzy control rules}

This procedure is focused on constructing a set of fuzzy IF-THEN rules. Here, we claim that the convergence is just at the beginning in case of a "VL" $e^{2}$ and a "VL" $\Delta e^{2}$, and thus a "VL" value for $p$ is used to speed up its convergence rate. On the other hand, the filter is assumed to operate in the steady-state status when $e^{2}$ and $\Delta e^{2}$ show " $\mathrm{S}$ ", and then " $\mathrm{S}$ " $p$ is adopted to lower its steady-state MSE. In particular, we may declare that a huge estimation error has occurred when $e^{2}$ is " $\mathrm{S}$ " and 
$\Delta e^{2}$ indicates "VL" and the "L" value of parameter $p$ is assigned to system in order to stabilize system performance.

\section{3) Fuzzy operators}

The fuzzified input variables are combined using the fuzzy "OR" operator, which selects the maximum value of the two, to obtain a single value. Subsequently, this is followed by the implication process, which defines the reshaping task of the consequent (THEN-part) of the fuzzy rule based on the antecedent (IF-part). A min (minimum) operation is generally employed to truncate the output fuzzy set for each rule. Since decisions are based on the testing of all of the rules in an FIS, the rules need to be combined in some manner in order to make a decision. Aggregation is the process by which the fuzzy sets that represent the outputs of each rule are combined into a single fuzzy set. The input of the aggregation process is the list of truncated output functions returned by the implication process for each rule. The output of the aggregation process is one fuzzy set for each output variable.

\section{4) Defuzzification processes}

The defuzzification process converts fuzzy control decision into non-fuzzy, control signals. These control signals are applied to adjust the variable of $p$ in order to improve convergence/tracking capability of the receiver. The crisp, physical control command is computed by the centroid-defuzzification method. The centroiddefuzzification output $p$ is calculated by [22]

$$
p(l+1)=\frac{\sum_{i=1}^{\Upsilon} p^{(i)}(l) \cdot m^{(i)}\left(p^{(i)}(l)\right)}{\sum_{i=1}^{\Upsilon} m^{(i)}\left(p^{(i)}(l)\right)},
$$

where the scalar $\gamma$ denotes the number of sections used for approximating the area under the aggregated MBFs, $p^{(i)}(l)$ is the value at the location used in approximating the area under the aggregated MBF, and $m^{(i)}\left(p^{(i)}\right.$ $(l)) \in[0,1]$ indicates the MBF value at location $p^{(i)}(l)$. The calculation of $p(l+1)$ in (18) returns the center of the area under the aggregated MBFs. It should be further emphasized that the determination of $\gamma$ is a trade-off between the system performance and the computational complexity of the FIS system. In order to alleviate the computational load in the centroid-defuzzification calculation of (18), fewer points $\Upsilon$ are preferred.

\section{Computational complexity analysis}

The calculation of the inverse of $\left(\mathrm{R}_{\mathbf{H}(l)} \mathrm{H}(l)+\frac{\beta}{\operatorname{SNR}} \mathrm{I}\right)$ and the product of $\mathbf{R}_{\mathbf{H}(l) \mathbf{H}(l)}\left(\mathbf{R}_{\mathbf{H}(l) \mathbf{H}(l)}+\frac{\beta}{\operatorname{SNR}} \mathrm{I}\right)^{-1}$ of the simplified WF estimator $\hat{\mathbf{H}}_{\mathrm{WF}}(l)$ in (8) costs $N^{3}+N^{2}$ complex multiplications if $\mathbf{R}_{\mathbf{H}(l) \mathbf{H}(l)}$ and SNR are assumed to be known beforehand or are set to fixed nominal values [23]. In what follows, the LS estimate of $\hat{\mathbf{H}}_{\mathrm{LS}}(l)=\mathbf{X}^{-1}(l) \mathrm{Y}(l)$ adopted in all three WF-based estimators requires $N$ multiplications The computational requirement of the product of $\mathbf{R}_{\mathbf{H}(l) \mathbf{H}(l)}\left(\mathbf{R}_{\mathbf{H}(l) \mathbf{H}(l)}+\frac{\beta}{\operatorname{SNR}} \mathbf{I}\right)^{-1}$ and $\hat{\mathbf{H}}_{\mathrm{LS}}(l)$ is $N^{2}$ multiplications. Therefore, the computational complexity of the simplified WF estimation in (8) expressed in terms of the number of complex multiplications is approximately given by $N^{3}+2 N^{2}+N$ for each OFDM block.

For the RR-WF estimator, the rank- $p$ approximation of the WF estimator in (13) can be re-expressed as a sum of rank-1 matrices as follows:

$$
\hat{\mathbf{H}}_{\mathrm{RR}-\mathrm{WF}}(l)=\left(\sum_{k=1}^{p} \delta_{k} u_{k} u_{k}^{\mathrm{H}}\right) \hat{\mathbf{H}}_{\mathrm{LS}}(l),
$$

where $\mathbf{u}_{k}$ denotes the $k$ th column vector in the matrix U. It should be noted that the vectors $\mathbf{u}_{k}$ for $k=1,2, \ldots$, $p$, can be tracked by means of the PASTd algorithm proposed in $[24,25]$ with a substantially reduced complexity of $2 N p$ for each OFDM block. The linear combination of $p$ vectors of length $N$ in (19) requires $N p$ multiplications. Thus, the RR-WF estimation of $\hat{\mathbf{H}}_{\mathrm{RR}-\mathrm{WF}}(l)$ accomplishes the total number of $3 N p+N$ complex multiplications, which is much less than that of the WF estimator. Remarkably, the complexity cost of the simplified WF estimator can be further reduced from $N^{3}+2 N^{2}+N$ to $3 N^{2}+N$ if the PASTd algorithm is applied to simplify Equation (12). Even though the complexity of the simplified WF estimator is st ill much higher than that of the rank- $p$ RR-WF estimator due to $p \ll N$.

The FIC RR-WF estimation with the time-varying filter rank $p(l)$ incurs a slighter computational complexity of $2 N p(l)$ in the tracking procedure of vectors $\mathbf{u}_{k}, k=1$, $2, \ldots, p(l)$, than the RR-WF scheme with the predetermined rank $p$, owing to the fact of $p(l)<p$. However, the additional computational load introduced by the (2-to1)-FIS, in terms of multiplications, is $\Upsilon+N+2$ at each OFDM block, in which the preparation of $e^{2}(l)$ requires $N+1$ multiplications and the centroid-defuzzification output process costs $\Upsilon+1$ multiplications. Furthermore, some special instructions (with a total of 24 lookups + 16 compares $+16 \Upsilon$ MAX operations) are required to perform the FIS, which come primarily from the fuzzification of two input variables (8 lookups), fuzzy OR operations (16 compares), fuzzy minimum implication (16 lookups), and aggregation of the output (16 $Y$ MAX operations). Fortunately, these operations can be done 
very efficiently in the latest range of DSPs, which provide single cycle multiply and add, table lookups and comparison instructions [26,27]. Thus, the FIC RR-WF estimation has the computational requirement of $3 \mathrm{~Np}(l)$ $+2 N+\Upsilon+2$ complex multiplications for the $l$ th OFDM block. Consequently, the saving of the FIC RRWF scheme in complexity over the RR-WF estimator can be achieved when the extra burden incurred by the (2-to-1)-FIS is lower than the advantage of $3 N(p-p(l))$ provided by the FIC-based rank reduction, i.e. $\Upsilon+N+$ $2<3 N(p-p(l))$. In addition, it should be further emphasized the fact that the RR-WF estimation with the use of a time-varying FIC rank possesses excellent channel dynamic tracking and adaptation capability over both the full-rank WF estimator and the RR-WF scheme with a fixed filter rank.

\section{Numerical results}

The channel estimation of MB-OFDM UWB systems can be performed by either adopting preamble training sequence or inserting pilot signals into each OFDM symbol. Here, we use a few pilots that are inserted into each OFDM symbol to estimate the channel frequency response (CFR) [5] in the interpolation-based channel estimators. In the piecewise linear interpolation algorithm, the estimation of the frequency-domain channel response located in between the pilots is performed by the linear interpolation, and the estimated pilot channel $\hat{H}_{p}\left(l, i_{n}\right)$ is updated by the LS estimation [9], given by

$$
\hat{H}_{p}\left(l, i_{n}\right)=\lambda \hat{H}_{p}\left(l-1, i_{n}\right)+(1-\lambda) \frac{Y_{p}\left(l, i_{n}\right)}{X_{p}\left(l, i_{n}\right)},
$$

where $\lambda$ is a forgetting factor $(0<\lambda<1)$. The parameters of computer simulations are mainly based on the Table 1 which summarizes the key parameters of the MB-OFDM UWB communication system. This MBOFDM UWB system uses an OFDM modulation scheme that utilizes 128 subcarriers per band, 122 of which are used to transmit the information. Of the 122 total subcarriers used, there are 100 used as data carriers, 12 used as pilot carriers, and 10 used as guard carriers. In our simulations, UWB channel models CM1, CM2, CM3, and CM4 are adopted. The channel model CM1 describes a line-of-sight (LOS) scenario when the distance between the transmitter and the receiver is less than $4 \mathrm{~m}$, whereas the CM2, CM3, and CM4 channel models represent the non-line-of-sight (NLOS) multipath channel environments with various delay dispersions [11]. Additionally, the $\left(e^{2}, \Delta e^{2}\right)$-FIS system with the $(4,4)$-partitioned regions to the fuzzy I/O domains is employed, due to its excellent performance and moderate complexity. Moreover, the MSE and the BER are used as the measures of their error performance related
Table 1 The parameters for MB-OFDM UWB systems in PHY

\begin{tabular}{|c|c|}
\hline Parameter & Value \\
\hline Modulation & QPSK \\
\hline Bandwidth & $528 \mathrm{MHz}$ \\
\hline$\sigma_{w}^{2}$ & 1 \\
\hline$\gamma^{\omega}$ & 4 \\
\hline$\lambda$ & $0.5(\mathrm{CM} 1, \mathrm{CM} 2, \mathrm{CM} 3), 0.3(\mathrm{CM} 4)$ \\
\hline$N_{h}$ & 5(CM1,CM2,CM3), 15(CM4) \\
\hline$N_{p}$ & 12 \\
\hline FFT Size $(N)$ & 128 \\
\hline Cyclic Prefix $(P)$ & 32 \\
\hline Pilot Spacing $\left(L=i_{n+1}-i_{n}, n \in\left[1, N_{p}\right]\right)$ & 8 \\
\hline $\mathrm{N}_{\mathrm{SD}}:$ Number of data carriers & 100 \\
\hline $\mathrm{N}_{\mathrm{SP}}$ : Number of pilot carriers & 12 \\
\hline $\mathrm{N}_{\mathrm{SG}}:$ Number of guard carriers & 10 \\
\hline 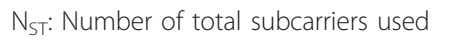 & $122\left(=\mathrm{N}_{\mathrm{SD}}+\mathrm{N}_{\mathrm{SP}}+\mathrm{N}_{\mathrm{SG}}\right)$ \\
\hline$\Delta_{F}:$ Subcarrier frequency spacing & 4.125 MHz(= 528 MHz/128) \\
\hline $\mathrm{T}_{\mathrm{FFT}}$ : IFFT/FFT period & $242.42 n s\left(=1 / \Delta_{F}\right)$ \\
\hline $\mathrm{T}_{\mathrm{CP}}$ : Cyclic prefix duration & $60.61 \mathrm{~ns}(=32 / 528 \mathrm{MHz})$ \\
\hline $\mathrm{T}_{\mathrm{Gl}}$ : Guard interval duration & $9.47 \mathrm{~ns}(=5 / 528 \mathrm{MHz})$ \\
\hline $\mathrm{T}_{\text {SYM: }}$ Symbol interval & $312.5 n s\left(=T_{C P}+T_{F F T}+T_{G I}\right)$ \\
\hline
\end{tabular}

to the implementation of the algorithms. The MSE is defined as the mean-squared error difference between the transfer function of transmission channel $H(l, k)$ and its estimate $\hat{H}(l, k)[10,28]$, as shown below

$$
\varepsilon \triangleq E\left\{|H(l, k)-\hat{H}(l, k)|^{2}\right\}, \quad k=0,1, \ldots, N-1 .
$$

Remarkably, the main difference between the MBOFDM UWB system and the common OFDM system is that the MB-OFDM UWB system uses a time-frequency kernel to specify the center frequency in the frequencyband group for the transmission of each OFDM symbol. When the specific sub-band signal transmission is identified by means of the TFCs, the transmitted symbols have no difference with the common OFDM systems. Hence, the proposed MB-OFDM UWB scheme can also be applied to perform signal detection in the OFDM systems.

In Figure 3, the MSE and the BER performance comparisons between the rank-reduction scheme based on the FIC RR-WF, the RR-WF, the piecewise linear, the Gaussian second-order, the cubicspline, the LS, and the full-rank WF schemes are evaluated in terms of SNR $(\mathrm{dB})$ in CM1. The proposed FIC RR-WF algorithm performs the fuzzy controlled filter-rank selection over both rank selection ranges $[2,8]$ and $[2,11]$. In both figures, it is observed that the performance of the cubicspline interpolation is better than those of the piecewise linear and the Gaussian second-order and is similar to 


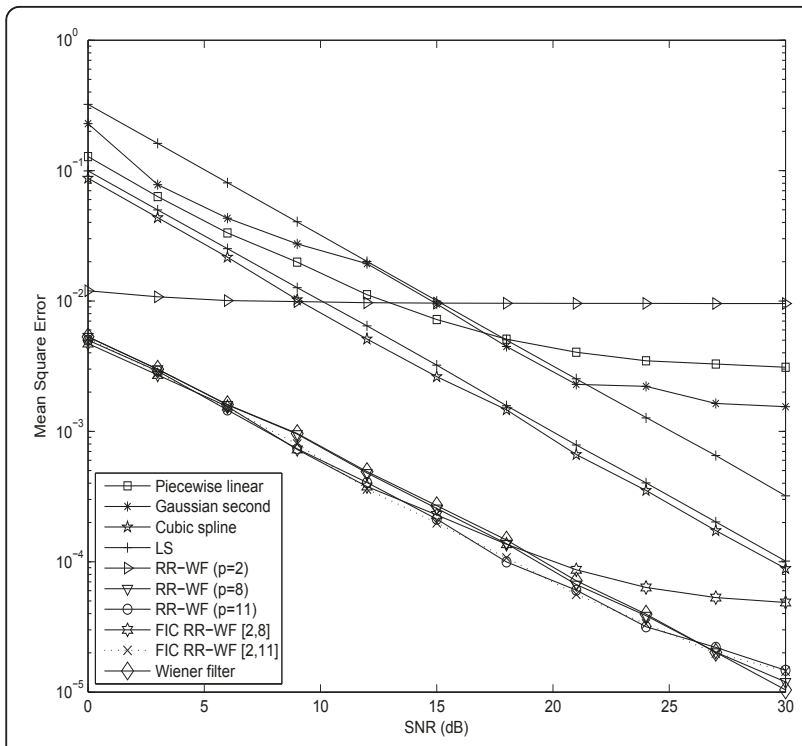

(a)

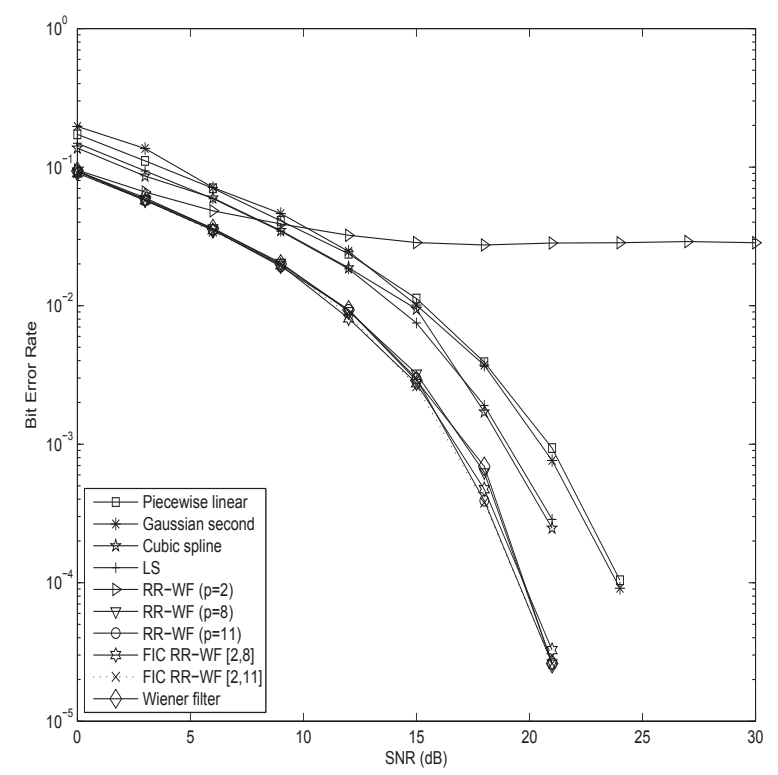

(b)

Figure 3 Performance comparisons of (a) the MSE and (b) the BER, between the FIC RR-WF, the RR-WF, the piecewise linear, the Gaussian second-order, the cubic-spline, the LS, and the WF in CM1.

that of the LS. This is reasonable because the higherorder interpolation scheme makes the given data points more smoothly. In addition, to evaluate how far the proposed FIC RR-WF scheme is from the optimal performance, we generalize the optimal estimator derived in

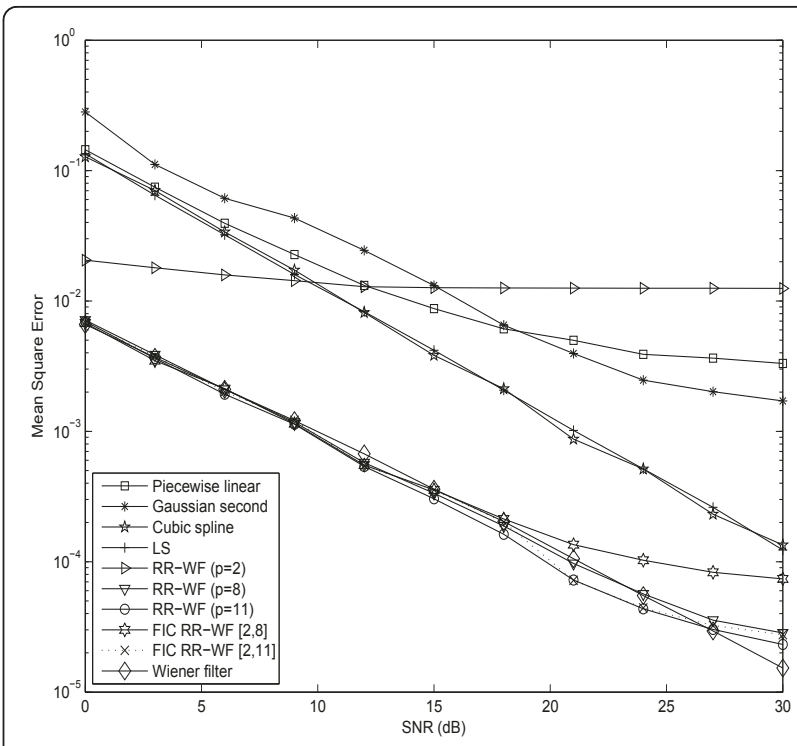

(a)

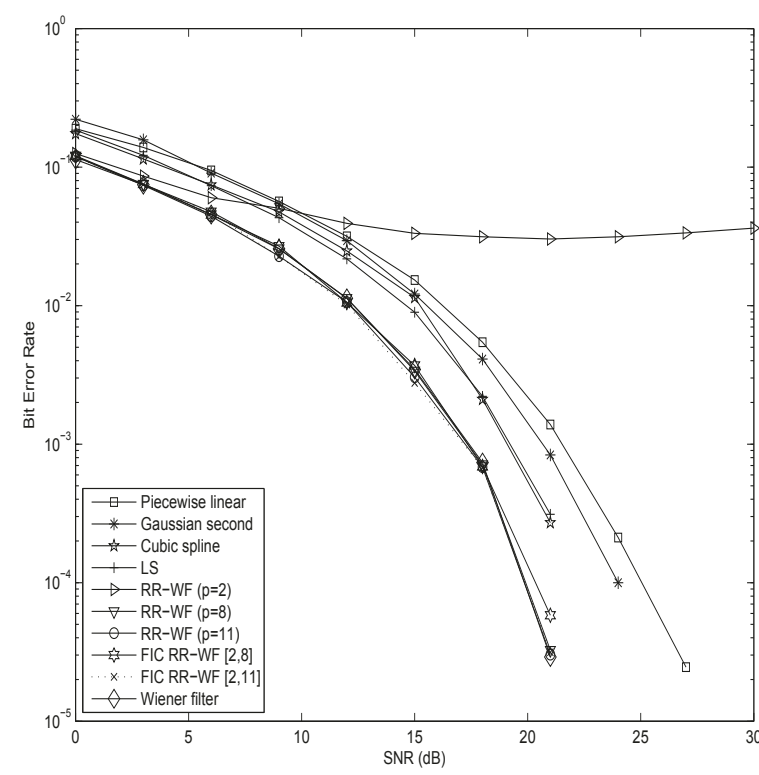

(b)

Figure 4 Performance comparisons of (a) the MSE and (b) the BER, between the FIC RR-WF, the RR-WF, the piecewise linear, the Gaussian second-order, the cubic-spline, the LS, and the WF in CM2.

[18], denoted as the Wiener filter. Hence, the performance of the WF could serve as the performance reference. As seen in Figure 3, the performance of the RRWF algorithm with the use of $p=8$ and the proposed FIC RR-WF scheme is close to that of the full-rank WF 


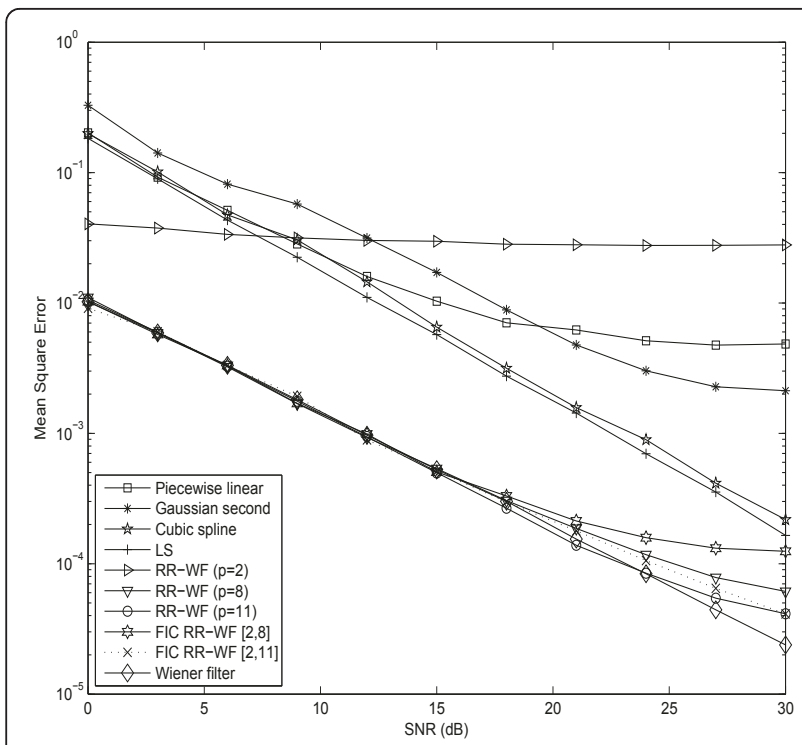

(a)

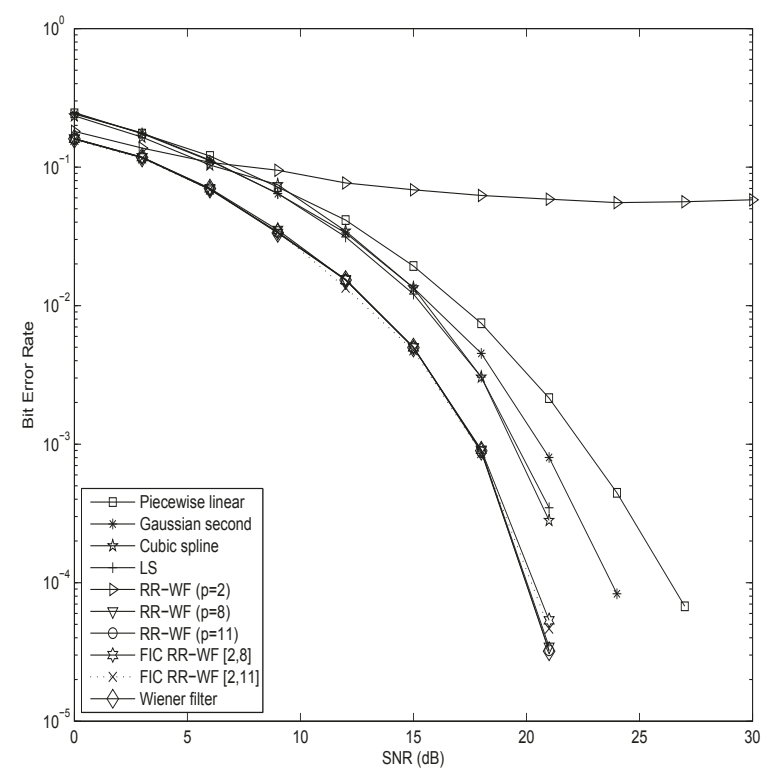

(b)

Figure 5 Performance comparisons of (a) the MSE and (b) the BER, between the FIC RR-WF, the RR-WF, the piecewise linear, the Gaussian second-order, the cubic-spline, the LS, and the WF in CM3.

estimator and is much better than those of other existing channel estimation schemes. However, the full-rank WF estimator is readily known to have more expensive computational cost than the RR-WF and the FIC RRWF channel estimators. Fortunately, the RR-WF

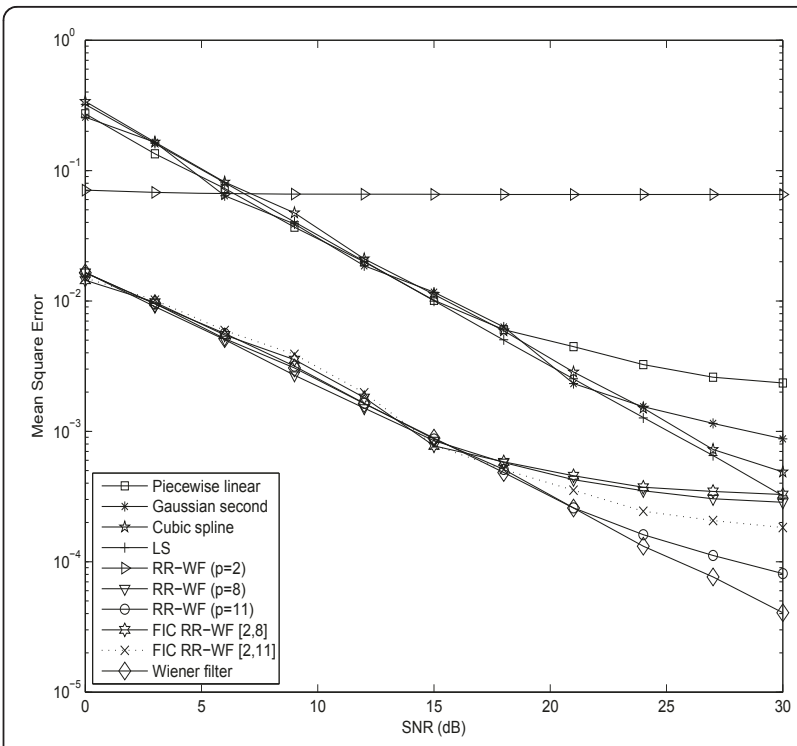

(a)

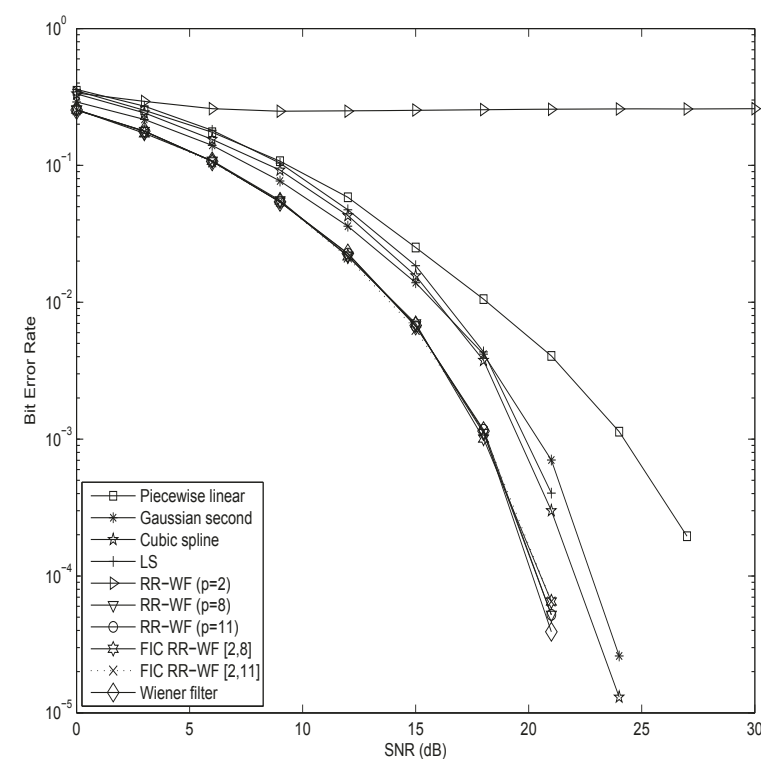

(b)

Figure 6 Performance comparisons of (a) the MSE and (b) the BER, between the FIC RR-WF, the RR-WF, the piecewise linear, the Gaussian second-order, the cubic-spline, the LS, and the WF in CM4.

estimation with the use of a time-varying FIC rank is capable of producing the BER performance similar to that of the full-rank WF channel estimator while accomplishing a substantial saving in complexity. In addition, results in the figure demonstrate that the FIC RR-WF 
with a larger rank selection range $[2,11]$ provides better performance than that of the FIC RR-WF with the selection range $[2,8]$, especially at the high SNR region. In Figures 4 and 5, the MSE and the BER performance comparisons between different channel estimation schemes are presented in terms of SNR for UWB channels CM2 and CM3, respectively. Results in Figures 4 and 5 demonstrate that similar MSE and BER performances to the CM1 in Figure 3 are achieved. Additionally, due to the stronger delay dispersion nature of both channels CM2 and CM3, the MSE and the BER performances degrade slightly as compared with that of the channel CM1. The MSE and the BER performances of those different channel estimation schemes with the use of the channel model CM4 are presented in Figure 6 in terms of SNR. It is observed from both figures that the MSE and the BER performances of all channel estimation schemes degrade dramatically as the channel model CM1 is switched to the CM4. This is because the time delay spread under the channel model CM4 is much more severe than that of the channel model CM1; therefore, the frequency selectivity between subcarriers
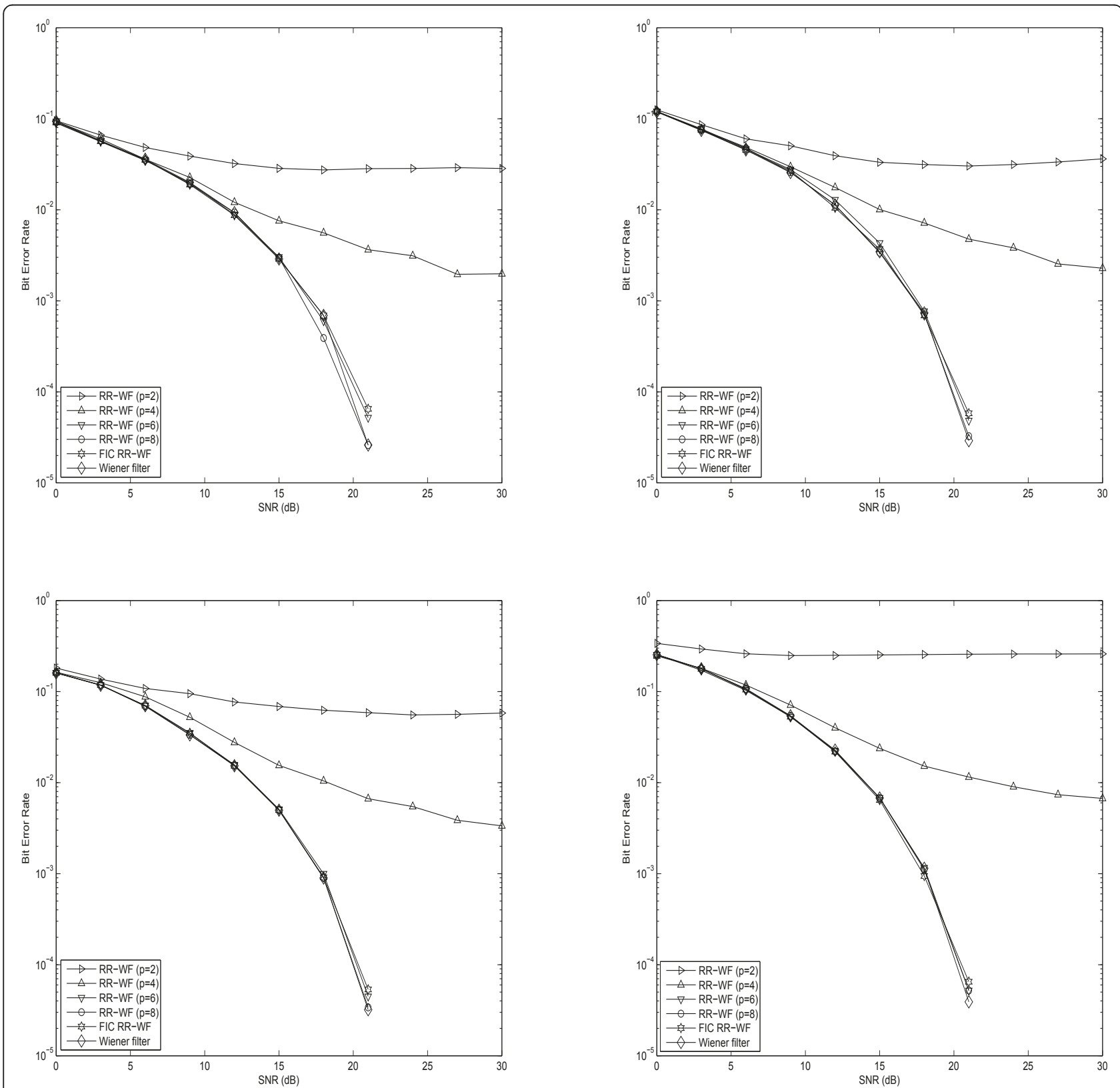

Figure 7 The BER performance comparisons between the RR-WF, the full-rank WF, and the FIC RR-WF in (upper-left) CM1, (upper-right) $\mathrm{CM} 2$, (lower-left) $\mathrm{CM} 3$, and (lower-right) CM4. 
of the CM4 is more serious than that of the CM1. However, it is seen from Figure 6 that the MSE and the BER performances of the RR-WF scheme with $p \geq 8$ are able to produce an identical BER performance level to the full-rank WF and superior than those of other interpolation-based channel estimation schemes.

In Figure 7, the BER performance is compared between the RR-WF, the full-rank WF, and the FIC RR-WF algorithms in terms of SNR for channel models CM1, CM2, CM3, and CM4, respectively. Results in Figure 7 demonstrate that the BER performance of all three WF-based schemes degrades as the UWB channel delay spreads are more severe. The proposed FIC RR-WF algorithm, which performs the fuzzy-logic filter-rank selection over the range of $[2,8]$, is able to take advantages of both small and large ranks in convergence and steady-state characteristics. The mean numbers of selected ranks achieved by the FIC RR-WF algorithm in 50 OFDM-frame calculations are, respectively, 5.19, 5.28, 5.41, and 5.66 for CM1, CM2, CM3, and CM4. The results in all figures show that the FIC RR-WF algorithm is able to accomplish a similar performance as the full-rank WF approach at a low rank (i.e. $p \leq 8)$. In other words, the FIC RR-WF algorithm is capable of achieving a substantial saving in complexity while maintaining a near full-rank WF performance.

\section{Conclusion}

In this paper, an adaptive FIC RR-WF channel estimation algorithm is proposed for the MB-OFDM UWB communication systems. This RR-WF-based algorithm employs an adaptive FIC filter rank in response to the time-invariant multipath fading channels. As a consequence, the FIC RR-WF channel estimation algorithm is capable of producing not only the BER performance similar to that of the full-rank WF channel estimator but also a substantial saving in complexity. Therefore, the proposed FIC RR-WF channel estimator is more feasible for applications in the MB-OFDM UWB wireless systems.

\section{Endnotes}

Four triangular MBFs with centroids of the very large $(\mathrm{VL})$, large $(\mathrm{L})$, medium $(\mathrm{M})$, and small $(\mathrm{S})$, respectively, are selected to cover the entire universe of discourse for variables $e^{2}, \Delta e^{2}$, and $p$.

\footnotetext{
Acknowledgements

This work was supported by Taiwan National Science Council under Grant NSC 97-2221-E-194-032.
}

\section{Competing interests}

The authors declare that they have no competing interests.
Received: 23 November 2010 Accepted: 18 September 2011 Published: 18 September 2011

\section{References}

1. H-J Park, M-J Kim, Y-J So, Y-H You, H-K Song, UWB communication system for home entertainment network. IEEE Trans Consum Electron. 49(2), 302-311 (2003). doi:10.1109/TCE.2003.1209518

2. Z Liang, S Zhu, S Wang, Space-time spreading based DS-UWB link scheme for wireless home entertainment networks. IEEE Trans Consum Elec. 52(3), 857-863 (2006). doi:10.1109/TCE.2006.1706481

3. A Batra, et al, MultiBand OFDM Physical Layer Proposal for IEEE802.15.3a. MultiBand OFDM Alliance SIG (2004)

4. A Batra, J Balakrishnan, GR Aiello, JR Foerster, A Dabak, Design of a multiband OFDM system for realistic UWB channel environments. IEEE Trans Microw Theory Tech. 52(9), 2123-2138 (2004). doi:10.1109/ TMTT.2004.834184

5. JJ van de Beek, O Edfors, M Sandell, SK Wilson, PO Börjesson, On channel estimation in OFDM systems, in Proceedings of IEEE 45th Veh. Technol. Conf., Chicago, Illinois, USA. 2, 815-819 (1995)

6. M Morelli, U Mengali, A comparison of pilot aided channel estimation methods for OFDM systems. IEEE Trans Signal Process. 49(12), 3065-3073 (2001). doi:10.1109/78.969514

7. R Negi, J Cioffi, Pilot tone selection for channel estimation in a mobile OFDM system. IEEE Trans Consum Electron. 44(3), 1122-1128 (1998). doi:10.1109/30.713244

8. H-Y Lin, C-C Hu, Y-F Chen, J-H Wen, An adaptive robust LMS employing fuzzy step size and partial update. IEEE Signal Process Lett. 12(8), 545-548 (2005)

9. P-Y Tsai, T-D Chiueh, Frequency-domain interpolation-based channel estimation in pilot-aided OFDM systems, in Proceedings of IEEE 59th Veh. Technol. Conf., Milan, Italy. 1, 420-424 (2004)

10. SG Kang, YM Ha, EK Joo, A comparative investigation on channel estimation algorithms for OFDM in mobile communications. IEEE Trans Broadcast. 49(2), 142-149 (2003). doi:10.1109/TBC.2003.810263

11. JR Foerster, et al, Channel Modeling Sub-Committee Report Final. IEEE Piscataway, NJ, P802.15-02/490r1-SG3a, Final Report (November 2003)

12. A Saleh, $R$ Valenzuela, A statistical model for indoor multipath propagation. IEEE J Select Areas Commun. 5(2), 128-137 (1987)

13. AF Molisch, JR Foerster, M Pendergrass, Channel models for ultrawideband personal area networks. IEEE Wireless Commun. 10(6), 14-21 (2003). doi:10.1109/MWC.2003.1265848

14. Y Mostofi, DC Cox, ICI mitigation for pilot-aided OFDM mobile systems. IEEE Trans Wireless Commun. 4(2), 765-774 (2005)

15. $\mathrm{H}-\mathrm{C} \mathrm{Wu}$, Analysis and characterization of intercarrier and interblock interferences for wireless mobile OFDM systems. IEEE Trans Broadcast. 52(2), 203-210 (2006). doi:10.1109/TBC.2006.872989

16. $\mathrm{H}-\mathrm{C}$ Wu, Y Wu, Distributive pilot arrangement based on modified msequences for OFDM intercarrier interference estimation. IEEE Trans Wireless Commun. 6(5), 1605-1609 (2007)

17. X Huang, $\mathrm{H}-\mathrm{C}$ Wu, Robust and efficient intercarrier interference mitigation for OFDM systems in time-varying fading channels. IEEE Trans Veh Technol. 56(5), 2517-2528 (2007)

18. O Edfors, M Sandell, JJ van de Beek, SK Wilson, PO Börjesson, OFDM channel estimation by singular value decomposition. IEEE Trans Commun. 46(7), 931-939 (1998). doi:10.1109/26.701321

19. LA Zadeh, Fuzzy sets. Inf Contr. 8, 338-353 (1965). doi:10.1016/S0019-9958 (65) $90241-X$

20. W-S Gan, Designing a fuzzy step size LMS algorithm. IEEE Proc-Vis Image Signal Process. 144(5), 261-266 (1997). doi:10.1049/ip-vis:19971417

21. L-X Wang, JM Mendel, Fuzzy adaptive filters, with application to nonlinear channel equalization. IEEE Trans Fuzzy Syst. 1(5), 161-170 (1993)

22. V Kecman, Learning and Soft Computing: Support Vector Machines, Neural Networks, and Fuzzy Logic Models (MIT Press, Cambridge, MA, 2001)

23. M Noh, Y Lee, H Park, Low complexity LMMSE channel estimation for OFDM. IEE Proc- Commun. 153(5), 645-650 (2006). doi:10.1049/ipcom:20050026

24. B Yang, Projection approximation subspace tracking. IEEE Trans Signal Process. 43(1), 95-107 (1995). doi:10.1109/78.365290

25. B Yang, An extension of the PASTd algorithm to both rank and subspace tracking. IEEE Signal Process Lett. 2(9), 179-182 (1995). doi:10.1109/ 97.410547 
26. MJ Patyra, JL Grantner, K Koster, Digital fuzzy logic controller: design and implementation. IEEE Trans Fuzzy Syst. 4(4), 439-459 (1996). doi:10.1109/ 91.544304

27. A Costa, A De Gloria, P Faraboschi, A Pagni, G Rizzotto, Hardware solutions for fuzzy control. Proc IEEE. 83(3), 422-434 (1995). doi:10.1109/5.364488

28. B Yang, KB Letaief, RS Cheng, Z Cao, Channel estimation for OFDM transmission in multipath fading channels based on parametric channel modeling. IEEE Trans Commun. 49(3), 467-479 (2001). doi:10.1109/ 26.911454

doi:10.1186/1687-6180-2011-64

Cite this article as: $\mathrm{Hu}$ and Lee: Adaptive low-rank channel estimation for multi-band OFDM ultra-wideband communications. EURASIP Journal on Advances in Signal Processing 2011 2011:64.

\section{Submit your manuscript to a SpringerOpen ${ }^{\circ}$ journal and benefit from:}

- Convenient online submission

- Rigorous peer review

- Immediate publication on acceptance

- Open access: articles freely available online

- High visibility within the field

- Retaining the copyright to your article

Submit your next manuscript at $\gg$ springeropen.com 